\title{
基于 STEAM 教育模式下无人机课程的研究
}

\section{Research on UAV Curriculum Based on STEAM Education Mode 林坤将 史蕾雨 苏敏华 林绮丹 丁群}

\author{
Kunjiang Lin Leiyu Shi Minhua Su Qidan Lin Qun Ding
}

华南农业大学珠江学院

中国·广东 广州 510000

Zhujiang College Of South China Agricultural University, SCAU(ZJ), Guangzhou, Guangdong, 510000, China

基金项目:广东省“创新强校工程”专项 建设项目资助和华南农业大学珠江学 院经费资助 “Steam”+“无人机”教育 【201912623008S】
【摘 要】论文结合 STEAM 教育模式对无人机课程进行研究, 以心理学家皮亚杰的认知 发展理论为主要指导思想, 对无人机课程进行级别划分, 采用内容分析法, 结合 PBL、创客 教育、MOOC 等新教育模式, 对各级无人机课程的设计理念、课程结构及教学方法进行研 究,运用 STEAM 教学方式加强课程的渗透,促使信息技术教学得到更好的发展,填补中国 在无人机课程的空缺, 为国家改革发展青少年科普教育的教学模式和方式提供参考示例。

【Abstract】Based on STEAM education model, this paper studies the UAV curriculum. With Piaget's cognitive development theory as the main guiding ideology, this paper classifies the UAV curriculum into different levels, uses content analysis method, combines with new education models such as PBL, Creator Education, MOOC, and so on. The design concept and curriculum of UAV curriculum at all levels are discussed. Structures and teaching methods are studied, and STEAM teaching method is used to strengthen the infiltration of courses, promote the better development of information technology teaching, fill the vacancies of domestic UAV courses, and provide reference examples for the national reform and development of the teaching mode and mode of popular science education for teenagers

【关键词】STEAM 教育; 无人机课程; 教学模式

【Keywords \STEAM education; unmanned aerial vehicle course; teaching model 【DOI】10.36012/sde.v2i1.760

\section{1 引言}

STEAM 教育指的是融合科学、技术、工程、艺术以及数学 于一体的综合性教育。该理念最初兴起于杜威、皮亚杰、维果 茨基等教育家以及心理学家的理论。除了倡导学科间的整合, 促进学生的自主学习, 强调合作探究, STEAM 教育还注重学 科知识与日常生活的联系。在 STEAM 理念的指导下,教师需 要鼓励学生拓展思维空间,培养创新精神。随着中国国力的增 强, 居民可支配收人不断提高, 人们的生活质量不断改善, 人 们逐渐把注意力放到子女的教育上来。在不断涌现的各种新 理念与新技术中, STEAM 教育理念掀起了教育体系改革的新 浪潮,逐渐成为当下教育体系改革和创新话题。由于人们对于 高质量教育的需求不断增加, 社会对综合型人才的需求不断 增加, 中国已有少数学校尝试开展与 STEAM 主题相关的课 程活动, 然而, 大部分学校仍然对 STEAM 教育缺乏正确并清 晰的认识。
中国的传统教育主要采纳的教学方式为教师讲授法, 由 任课教师在课堂上向学生讲授完所有理论知识之后, 再进入 实践阶段。然而, 这种教学方式实践于探究性课程是不合适 的。这种教学方式的消极影响是:学生缺乏对研究对象的感性 认识, 认为所学理论知识与实际生活脱节, 没有实际作用, 从 而缺乏学习主动以及积极性。因此, 本文将从 STEAM 教育的 背景以及在中国实施现状出发, 将无人机课程教学与其进行 结合研究, 强调注重通过科学教育实践动手操作、课程设置等 方式培养学生兴趣的同时，充分利用无人机技术学科交叉性 和实践操作性,将科学过程、技术素养、工程设计、艺术情操和 数学素养融合在各个教学情境中, 并结合不同年龄段学生的 特征给予适当的教学方法, 在 STEAM 教育模式的引领下通过 有针对性的教学设想来实现无人机课程的传授和实际操作。

随着中国互联网技术以及人工智能等新兴行业的迅猛发 展,未来国家想要傲立于世界民族之林,谋求更好的发展, 必 须依靠具有创新精神的科技人才。因此,培养儿童 STEAM 素 
教育理论与研究 Educational Theory and Research

养的任务是当务之急,不可忽视。基于 STEAM 教育模式下无 人机课程的教学不仅有利于发展儿童跨学科研究能力和培养 创新精神, 也促进了 STEAM 课程与科学课程过渡, 同时为儿 童的终身学习和发展奠定了坚实基础。

\section{STEAM 教育、无人机课程的背景和现状}

\subsection{STEAM 教育的发展背景和现状}

1986 年, 美国国家科学基金会发布的《本科的科学、数学 和工程教育》报告中提出了“科学、数学、工程和技术教育集 成”的纲领性建议, 目的是“使美国下一代成为世界科学和技 术领导者”, 这被视为提倡 STEM 教育的开端。进入 21 世纪, STEAM 教育的创始人 Yakman 把 “艺术” 作为重要的人文因 素与 STEM 相融合 ${ }^{[1]}$, 以弥补 STEM 教育缺乏趣味性与艺术性 的弊端。在 STEM 教育完善发展而来的 STEAM 教育, 旨在培 养学生综合素质及综合型创新人才, 有利于促进社会的进一 步发展。2011 年和 2013 年, 美国先后公布了《K-12 科学教 育框架》和《新一代科学教育标准》, 将科学与工程实践、跨学 科概念和核心概念紧密整合，并将 STEM 教育纳入国家标 准,这引起了国际教育界的关注 ${ }^{[2]}$ 。

在理论研究方面,在中国, 关于 STEAM 教育的学术探讨 仍未足够深人, 对 STEAM 教育的研究与世界前沿水平仍有 差距。而在实际教学中, 课堂缺乏培养学生与 STEAM 教育相 对应的软实力,如“演讲、交际、创新”等能力。并且,在中国利 用 STEAM 教育作为指导, 并贯彻与课堂教学的主体以培训 机构为主, 在学校课堂中却难以发现与 STEAM 教育理念紧 密相连的课堂活动, 且依旧采用传统的教授教学法, 未形成规 范的 STEAM 教学模式。

\subsection{STEAM 无人机课程的发展背景和现状}

随着科学技术的不断发展，无人机应用的领域越来越广
泛, 因此无人机行业呈现爆炸式增长, 这恰恰也是市场需求不 断增长所导致的。比如, GPS、陀螺仪等多种技术都能广泛应 用于智能设备中, 使人们的生活更加便利, 然而中国对于无人 机技术所提供的教育与培训并不多, 而以中小学生为对象的 STEAM 无人机技术科普教育更是少之又少。同时, 目前中国 高校所提供的无人机课程往往只局限于某一学科的应用。

2015 年,主要从事无人机教育事业的中科大智正式发布 了一款面向中小学生的无人机技术科普课程。这款具有自主 知识产权的课程, 在中国首次把无人机技术与科普教育结合 起来, 使中国的中小学生得以接触并学习到无人机技术。

无人机技术的快速发展与不断进步吸引着大众的目光, 人们对无人机教育行业的兴趣也愈加浓厚。“中国制造 2025 ” 的推行, 也给有在无人机行业有特殊才能的人提供了 展示自我的机会。然而, 与中国传统教育模式相差甚远的 STEAM 教育给教师提出了更高的要求, 需要教师注重学科 间的融合, 并提高学生的全面素养, 同时也要重视学习过 程, 强调时间。

\section{STEAM 教育模式下的无人机课程建构}

无人机教育行业越来越受关注, 但是相应的课程还不够完 善, 因此, 本文设想了 STEAM 教育模式下无人机的课程, 为无 人机教育行业的发展提供参考。下面将结合皮亚杰的认知发展 理论, 把无人机课程分为初级、中级、高级 3 个层次进行教学。

\section{1 “LBL+ 创客教育 $+K W L ”$ 模式的初级课程}

“LBL+ 创客教育 $+\mathrm{KWL}$ ”模式的初级课程如表 1 所示。

初级课程的适用年龄段为 7 10 岁的儿童, 本阶段的儿童 符合皮亚杰认知理论的具体运算阶段的特征, 这一阶段儿童 的认知结构已发生了重组和改善, 思维具有一定的弹性, 能凭 借具体事物或从具体事物中获得的表象进行逻辑思维和群集

表 1 无人机初级课程

\begin{tabular}{|c|c|c|c|}
\hline 模式 & LBL & 创客教育 & KWL \\
\hline $\begin{array}{l}\text { 模式 } \\
\text { 特点 }\end{array}$ & 教师主导 & $\begin{array}{l}\text { 培养动手能力 } \\
\text { 跨学科解决问题 }\end{array}$ & $\begin{array}{l}\text { 批判性思维 } \\
\text { 以学生为中心 } \\
\text { 以教师为主导 }\end{array}$ \\
\hline $\begin{array}{l}\text { 学习 } \\
\text { 方式 }\end{array}$ & $\begin{array}{c}\text { 目标驱动 } \\
\text { 教科书驱动 } \\
\text { 依赖性解决问题 }\end{array}$ & $\begin{array}{l}\text { 创新驱动 } \\
\text { 自主驱动 } \\
\text { 多元化解决问题 }\end{array}$ & $\begin{array}{c}\text { 引导驱动 } \\
\text { 自主合作探究解决问题 }\end{array}$ \\
\hline $\begin{array}{l}\text { 模式 } \\
\text { 评价 }\end{array}$ & $\begin{array}{c}\text { 全面 } \\
\text { 系统性强 } \\
\text { 信息量大 } \\
\text { 进展速度快 } \\
\text { 学科关联性弱 } \\
\text { 忽视学生能力培养 }\end{array}$ & $\begin{array}{l}\text { 协同性强 } \\
\text { “做中学” }\end{array}$ & $\begin{array}{l}\text { 自主反思 } \\
\text { 自我评价 } \\
\text { 培养习惯 } \\
\text { 课后巩固 } \\
\text { 终身受益 }\end{array}$ \\
\hline $\begin{array}{l}\text { 课程 } \\
\text { 内容 }\end{array}$ & & 木、绘画并造 & 编程 \\
\hline
\end{tabular}


运算 ${ }^{[3]}$ 。儿童能够运用逻辑,思维解决具体问题, 但必须依赖实 物或者直观形象的支持进行思考，针对本阶段的儿童心智发 展特征设计了“LBL+创客教育 $+\mathrm{KWL}$ ”的教学模式, 既发挥了 教师的主导作用, 让儿童对无人机拥有最直观的认识, 也发挥 了学生的主体作用,激发他们对无人机的兴趣。

初级课程首先利用观影拓展的方式来介绍无人机的定义 和分类, 如让学生观看相应的航拍图片或视频,引出无人机是 一种不需要人驾驶的飞行器, 在各国的军事领域发挥重要作 用等概念。了解当前无人机的多种分类方式, 如按用途分类、 按飞行平台构型分类、按动力源分类等。此外, 课堂上还会科 普拓展无人机的飞行原理、构造、历史等相关知识。在授课过 程中,会结合多媒体手段,播放相关的无人机图片、动画、视频 等,使学生全方位了解无人机, 发挥教师的启蒙作用。

课程除了采用 LBL, 还采取创客教育来辅助教学。创客教 育倡导学生勤动手, 鼓励学生发明工艺、分享创意点子, 贯彻 “做中学”思想, 培养跨学科解决问题的能力以及团队协作能 力。安排学生动手拼装无人机积木, 然后分享创意, 互相交流 拼装过程及心得, 让学生在创造中获得知识, 同时锻炼他们的 动手、沟通、协作能力等。最后教师进行必要的总结, 让学生推 选出最有创意的无人机积木, 给予奖励。

课程利用学生的直觉思维可进行“纸上涂鸦”, 人的直觉思 维往往是在边做边想时发生, 有直观实践性, 因此利用学生的直 觉对无人机进行绘画, 培养学生的审美以及洞察力, 渗透了 STEAM 教育的“Arts”理念。而“实物创造”环节, 教师需提前布置 学生搜集造物材料, 鼓励废物利用, 课堂上安排学生进行造物, 并让学生介绍自己物品的创意创新点, 最后老师主持学生进行 作品展览。在“造物”过程中, 通过创客教育增强学生的创新思维 能力和创造力,使学生对无人机有新的思考和更全面的了解。
课程后半段进入飞行教学环节, 先利用遥控器操控计算 机画面中的虚拟无人机飞行, 熟悉空间三维方向的运动, 了解 飞行的操作原理,让学生身临其境, 为操控真实无人机奠定基 础。在学生对操控无人机有一定的认识后,进行基本的真机飞 行, 此过程学生需要掌握美国手遥控器的摇杆模式, 学会起 飞、降落、定高移动、方向控制等基本飞行操作, 知晓飞行时的 注意事项以及电池的适用和维护 ${ }^{[4]}$ 。

飞行实操过后, 运用 Tello Edu 学习简单编程, 它是一款 独一无二的教育编程无人机, Tello Edu 结合编程和无人机两 方面, 让学生在游戏中循序渐进地学习编程, 还可以检查自己 的代码逻辑正确与否, 实现自主学习, 激发学习兴趣。在课程 中, 教师除了锻炼学生的逻辑思维能力外, 更注重培养学生的 认知能力。编程教学融合了 STEAM 教育理念, 进行多学科交 互,提升学生综合运用能力。

本课程为贯彻自主、合作、探究的学习方式,培养学生的 批判性思维,增强学生的自我反思和自我评价能力, 在每节课 结束之后, 还会采用 KWL 学习表格让学生对课堂知识进行 回顾和反思。 $\mathrm{K}$ (What I know) 是已知, W(What I want to know) 是想知, $\mathrm{L}$ (What I learned ) 是学知 ${ }^{[5]}$, 这三者的结合可以让学 生养成自我评价的习惯, 对自己的学习建立元认知, 从而使学 生终身受益。

无人机课程融汇了多学科内容, 因此, 在课程的最后, 会 安排复习回顾课, 利用 KWL 教学法, 帮助学生理解学习内容, 增强学习的有效性。以上就是无人机初级课程的全部内容。

\section{2 “引导一一发现式课堂 $+T B L+K W L ”$ 模式的} 中级课程

“引导——发现式课堂 + TBL + KWL” 模式的中级课程如 表 2 所示。

表 2 无人机中级课程

\begin{tabular}{|c|c|c|c|}
\hline 模式 & 引导一发现式课堂 & TBL & KWL \\
\hline $\begin{array}{l}\text { 模式 } \\
\text { 特点 }\end{array}$ & $\begin{array}{c}\text { 以问题解决为中心 } \\
\text { 以培养学生的探求新知、概括归纳能力为教学目标 }\end{array}$ & $\begin{array}{c}\text { 以团队为基础学习 } \\
\text { 辩证教学模式 } \\
\text { 培养协作精神,注重创造性、灵活 } \\
\text { 实践性 }\end{array}$ & $\begin{array}{l}\text { 批判性思维 } \\
\text { 以学生为中心 } \\
\text { 以教师为主导 }\end{array}$ \\
\hline 学习方式 & $\begin{array}{l}\text { 问题驱动 } \\
\text { 理论导向 } \\
\text { 引导发现 }\end{array}$ & $\begin{array}{c}\text { 组建小组 } \\
\text { 才队讨论学习 }\end{array}$ & $\begin{array}{c}\text { 引导驱动 } \\
\text { 自主合作探究解决问题 }\end{array}$ \\
\hline $\begin{array}{l}\text { 模式 } \\
\text { 评价 }\end{array}$ & $\begin{array}{c}\text { 自我激发内在动机 } \\
\text { 获得解决问题的能力 } \\
\text { 利于学生记忆的保持 } \\
\text { 耗费时间 }\end{array}$ & $\begin{array}{l}\text { 培养才队合作和交际能力 } \\
\text { 理论知识掌握不透彻 } \\
\text { 独立思考能力有待提高 }\end{array}$ & $\begin{array}{l}\text { 自主反思 } \\
\text { 自我评价 } \\
\text { 培养习惯 } \\
\text { 课后巩固 } \\
\text { 终身受益 }\end{array}$ \\
\hline $\begin{array}{l}\text { 课程 } \\
\text { 内容 }\end{array}$ & 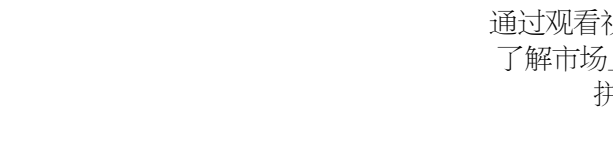 & $\begin{array}{l}\text { 频的方式,学习简单航拍知识 } \\
\text { 不同的机型并进行对比学习 } \\
\text { 装无人机并进行飞行 } \\
\text { 无人机高级编程 }\end{array}$ & \\
\hline
\end{tabular}




\section{教育理论与研究 Educational Theory and Research}

借鉴皮亚杰认知发展理论形式运算阶段的特征, 11 14岁 的青少年儿童抽象思维发展和完善 ${ }^{[0]}$, 能从多种维度对抽象 的性质进行思维, 但在实际生活中仍会刻板遵守规则[7], 针对 本阶段的儿童心智发展特征, 相对应的设计中级课程的教学 模式为“引导一发现式课堂+TBL+KWL”。此课堂教学以学 生主体、教师引导的方式来培养学生的积极主动性, 培养团队 合作精神,并学会总结反思。

中级课程先让学生观看视频, 了解什么是光圈、快门和 ISO, 怎样构图, 然后老师举例让概念形象具体化。比如, 若把 光圈当作一扇门, 光圈越大, 门开的越大, 进来的光线就多; 若 把快门当作开门和关门,当快门的速度是 $1 / 1250$ 时表示门只 开了 $1 / 1250$ 秒, 同理快门速度是 1 时门开了 1 秒, 显然 1 秒 进来的光线比 1/1250 秒进来的多; ISO 即感光度，感光度越 大, 照片越亮, 白天一般 ISO100 或 200, 夜晚根据光线环境相 应提高 ISO 数值; 无人机航拍取景构图灵活自由,如三分构图 一般适合突出多个景物, 居中构图一般适合单个主体等。这一 阶段的学习丰富学生对航拍知识科学的认识, 提高了学生的 审美能力, 是 STEAM 中“Science”和“Arts”的结合。

课程贯穿采用 TBL(以团队为基础的学习方式), 由教师 根据每个学生的特点组建学习小组, 提前确定无人机教学内 容供学生进行课前准备, 然后在课堂教学中侧重于才犾队共同 协作完成无人机拼装并进行飞行，让学生在课堂上及时发现 问题并快速解决。本阶段学生需以才模式制作一架无人机 (以小型四旋翼飞行器为例), 此过程中, 学生需要了解组装飞 机的使用材料、组装顺序、CC3D 的使用方法以及在组装中的 注意事项等。无人机组装完毕后,学生需要进行试飞,试飞包 括熟悉美国手遥控器的摇杆模式、飞行操作步骤、空中悬停操 作、飞行注意事项等 ${ }^{\left[{ }^{[}\right]}$。这一环节的学习不但可以培养学生动 手协调能力、空间想象能力、逻辑思维能力, 而且使学生各方 面能力在潜移默化中得到提升, 也学到更多无人机知识。
无人机组装环节过后, 进行无人机航拍飞行知识的学习, 先安排学生在课室观看无人机航拍飞行基础介绍视频, 然后 老师再进行讲授基础航拍飞行理论知识及操作过程中需要注 意的事项,并回顾前面所学的航拍知识, 两者结合让学生对无 人机航拍飞行有个大概的了解和掌握。此外, 还会向学生普及 无人航拍机起飞前的准备工作、升空和降落的注意事项、意外 的处理技能以及航拍机的保养知识。然后在户外进行实际航 拍飞行学习, 要求学生学以致用, 能根据实际情况调节光圈、 快门、ISO 的数值、构图等, 来完成图片的拍摄。

但学生学习知识, 难免会出现对理论知识掌握不透彻, 缺 乏独立思考的现象。因此, 中级课程继续采用“KWL 学习表 格” 的模式, 在每节课结束时, 预留时间让学生对本节课进行 总结反思, 帮助学生巩固知识, 发现不足。在本课程的后半段 进行无人机高级编程的应用教学, 先让学生回顾初级编程知 识, 再利用 Tello Edu 让学生轻松学习 Scratch 等编程语言, 掌握如何利用支持命令和数据交互以及编写代码来指挥多台 Tello Edu 编队飞行, 使得编程生动有趣。掌握并熟练这个应 用的板块及使用:第一是编程人门游戏, 利用积木编程控制飞 行器闯关, 巩固初级课程的编程知识, 也可进行真机编程操 控; 第二是 “遥控” 手柄, 可与无人机进行 wifi 链接, 遥控飞行; 第三是利用积木编写程序控制无人机。另外, 在课程的最后会 采用 KWL 教学法让学生对整个中级课程进行回顾和总结。

无人机课程运用“引导——发现式” 教学引导学生发现问 题, 运用 TBL 教学方式以团队为中心进行创造性、灵活实践 性学习, 运用 KWL 教学方式[9]进行总结反思学习, 这样就完 成了整个新知识获得的环节, 体现 STEAM 教育中注重整体 性的特点。综上, 无人机课中级课程设计结合 3 种教学方法, 体现了STEAM 教育的核心。

\section{3 “PBL+MOOC” 模式的高级课程}

“PBL+MOOC” 模式的高级课程如表 3 所示。

表 3 无人机高级课程

\begin{tabular}{|c|c|c|}
\hline 模式 & PBL & MOOC \\
\hline 模式特点 & $\begin{array}{c}\text { 以问题为导向学习 } \\
\text { 以学生为主导 } \\
\text { 问题基于真实情景 } \\
\text { 各种学科知识的交叉 }\end{array}$ & $\begin{array}{c}\text { 课程资源丰富 } \\
\text { 突破时间空间限制 } \\
\text { 开放性 }\end{array}$ \\
\hline 学习方式 & $\begin{array}{c}\text { 项目问题驱动 } \\
\text { 自主合作探究解决问题 } \\
\end{array}$ & $\begin{array}{c}\text { 利用线上资源 } \\
\text { 扩充学生的知识面 }\end{array}$ \\
\hline 模式评价 & $\begin{array}{c}\text { 提高解决实际问题的能力 } \\
\text { 培养团队合作和交际能力 } \\
\text { 组员间配合度不够 } \\
\text { 学生基础学科能力薄弱 }\end{array}$ & $\begin{array}{l}\text { 课前扩充 } \\
\text { 课后巩固 }\end{array}$ \\
\hline 课程内容 & \multicolumn{2}{|c|}{$\begin{array}{c}\text { 深化学习无人机理论知识 } \\
\text { 合作完成无人机高级编程 } \\
\text { 合作完成航拍作品 } \\
\text { 设计并完成团队任务 }\end{array}$} \\
\hline
\end{tabular}


根据儿童认知发展特征, 11 18 岁的年龄阶段中青少年 个体推理能力得到提高, 他们能从多种维度对抽象的性质进 行思维, 能够进行假设性思维, 采用逻辑推理、归纳或演绎的 方式来解决问题,其思维发展已接近成人的水平。因此, 采用 项目式学习的模式进行探究有助于青少年自身逻辑思维能 力、跨学科学习能力的培养和发展。“以问题为导向的学习” $(\mathrm{PBL})+\mathrm{MOOC}$ 线上资源拓展知识面的教学模式将贯穿于 STEAM 教育下无人机高级课程。

美国的 Barrows 教授于 1969 年首创 PBL(Problem-Based Learning) 教学方法, 这是一种以问题为导向、以学生为中心的 教育方式, 本文称之为项目式学习。项目式学习推崇学生自主 探究、团队协作, 以解决复杂、有意义的现实问题或运作实际 项目为目标; 从中学习隐含在问题、项目背后的学科知识, 进 而培养学生独立思考自主学习的能力和解决实际问题所需的 软实力。无人机高级课程的项目化可以引导学生主动投入学 习,对无人机理论概念、构造原理、飞行等理论知识在实际应 用的过程中形成更深度的理解。

无人机高级课程的就读学员具备基础的无人机操控、组 装和飞行等基础的知识, 而进一步深化学习无人机的理论知 识是高级课程的目标之一。由于无人机理论知识涵盖力学、空 气动力学、数学等多维度和跨学科领域的知识, 如何将抽象的 知识拆分、理解、内化再关联将贯穿于整个教学环节。在项目 式学习中, 学生由知识的被动接受者转为主动学习者、合作者 和研究者,成为此种教学模式的主体。而教师的角色转变为教 学组织者、资源提供者、学生能力发展的促进者和自主学习的 指导者。在项目设计时不仅需要关注学生的学习目标, 同时也 需要满足重点知识的学习和软技能的培养、项目目标的可完 成性、持续性的探究、项目的真实性、学生的发言权及选择权、 评论与修正、项目成果的公开展示这 7 项核心要素。

以下是无人机项目式学习课程设计案例一以 7 分钟视 频项目为例:

第一步: 教师提前进行相关知识的铺垫, 确保项目围绕所 学知识展开应用,确立航拍视频以自然为方向, 接着学生展开 头脑风暴,进而确立小组的视频主题。学生在界定问题、收集 资料、分析资料、建立假设、比较不同的方案和解决面临的问 题等过程中,教师需给予评估意见、项目指导等帮助。

第二步: 每个小组根据选定的视频主题进行相关剧情设 计、视频剪辑等相关内容, 课堂上汇报小组项目进度, 讨论如 何解决项目进行过程中遇到的难题, 教师进行指导以及知识
拓展, 对每位学生做出阶段性和总结性的评估。

第三步：项目成果汇报，教师筹备线上线下项目展示平 台,邀请父母或其他观众一同参与成果展示环节,观看视频作 品并进行评比,对学生给予肯定和奖励。

综上所述，围绕 STEAM 理念设计的高级无人机教学课 程采用 PBL 项目式学习教学法为主, 借助 MOOC 或其他资 源进行知识背景引入、拓展的方式去引导学生自主设计并完 成项目。该课程通过将学习目标项目化、问题化的教学方式, 最大限度地激发学生投入学习无人机知识、参与设计项目的 热情, 达到提高学生跨学科学习、批判性思考和解决实际问 题的能力。

\section{4 结语}

无人机课程有效合理融入了 STEAM 教育理念, 是培养中 国教育发展要求的新型人才的必然趋势。本文以皮亚杰认知 发展理论为指导, 构建了 STEAM 教育模式下无人机课程的 新蓝图, 以期为 STEAM 教育模式下无人机课程的研究与实 践提供新的参考和借鉴。无人机行业正处于发展上升期,相应 人才储备不足, STEAM 无人机教育课程为企业储备人才, 且 随着互联网和人工智能等科学技术迅猛发展, 未来国家发展 依靠具有创新精神的科技人才, 培养青少年儿童 STEAM 素 养变得至关重要。

\section{参考文献}

[1]崔鸿,朱家华张秀红.基于项目的 STEAM 学习探析:核心素养 的视角[J].华东师范大学学报(教育科学版),2017,35(4):54-61.

[2]陈琦,刘儒德.当代教育心理学[M].北京: 北京师范大学出版社, 2007.

[3] 崔胜民.小创客玩转无人机[M].北京: 化学工业出版社,2018.

[4]卢海,陈怡雯. 设计我们的海绵社区 $[\mathrm{M}]$.上海:上海教育出版社, 2018.

[5]张潮.教育心理学[M].北京:高等教育出版社,2017,164.

[6]单俊业,柳嘉惠.道德量化评价刍议[J].江苏理工学院学报, 2015,21(3):118-122.

[7]高桥隆雄.青少年无人机制作指南: STEAM\& 创客教育实践 课程[M].陈刚,译.北京:北京科学技术出版社,2017.

[8]Capiow JA, Donaldson JF, Kardash C. Learning in Problentbased Leaming Medical Curriculum: Students Conceptions[J].Med Edc,1997(31): 440-441.

[9]戴淑芳,孙艺平,刘佳,等.多种教学方法在医学机能实验教学中 的灵活运用 [J].实验室科学,2013,16(2):82-84. 